\title{
The effectiveness of a hospital-based intervention for patients with substance-use problems in the Western Cape
}

\author{
Katherine Sorsdahl, Dan J Stein, Lize Weich, David Fourie, Bronwyn Myers
}

District hospitals regularly experience a high incidence of substanceuse disorders, but rarely provide interventions. We describe the effectiveness of an intervention developed and implemented by a Western Cape hospital. Patients with probable substance use were referred to an on-site social worker for an alcohol, smoking and substance involvement screening test (ASSIST), a brief motivational intervention and referral to specialist care. At the 3-month followup, the ASSIST was re-administered telephonically. An intervention was received by 127 patients. A significant reduction in substance use was reported in 92 patients who completed a 3-month followup evaluation $(p<0.001)$. Of the 60 patients referred to further care, half entered treatment. We conclude that, with minimal resourcing, it is feasible to administer a brief substance-use intervention for patients attending district hospitals.

S Afr Med J 2012;102(7):634-635.
Substance-use disorders are a major global public health concern. The recent South African stress and health study (SASH) indicated a high lifetime prevalence (13.3\%) and early onset (21 years) of such disorders, ${ }^{1}$ with significantly higher rates in the Western Cape compared with other provinces. Substance-abuse treatment services are limited in the Western Cape, where existing services are overwhelmed by the demand for treatment. ${ }^{2}$

Increasing the range of treatment services is one way of improving access. ${ }^{2}$ The existing system relies heavily on provision of highthreshold treatment services by specialist providers; few lowthreshold early-intervention services are available at a primary healthcare level. This limits access to care as high-threshold services are costly. More cost-effective lower threshold services are preferable.

Evidence suggests that screening, brief intervention, and referral to treatment (SBIRT) is effective for addressing mild to moderate substance-related problems and is feasible to implement. ${ }^{3,4}$ However, there is little evidence for the effectiveness or feasibility of SBIRT for substance use in South Africa. We describe preliminary outcomes from a hospital-based SBIRT programme.

\section{Methods}

The SBIRT programme emerged from the realisation that substancerelated psychoses were observed in more than $80 \%$ of the 250 monthly patients seeking psychiatric services in G F Jooste Hospital (David Fourie, personal communication). A substance-abuse intervention model was developed by SANCA Western Cape, the Department of

Department of Psychiatry and Mental Health, University of Cape Town Katherine Sorsdahl, $\mathrm{PhD}$

Dan J Stein, MD, PhD

Bronwyn Myers, $\mathrm{PhD}$

Department of Psychiatry, University of Stellenbosch

Lize Weich, FCPsych

South African National Council on Alcoholism and Drug Dependence (SANCA), Tygerberg

David Fourie, DPhil

Alcohol and Drug Abuse Research Unit, Medical Research Council of South Africa Bronwyn Myers, PhD
Social Development, the Department of Health and Cape Town Drug Counselling Centre. Implementation of the model saw the initiation of a substance-abuse services centre at the hospital, staffed by a social worker, an auxiliary social worker and a research assistant.

Over a 7-month period, patients referred to the centre were given verbal and written information about the programme. A modified version of the alcohol, smoking and substance involvement screening test (ASSIST) was used to screen patients for substance use. ${ }^{5}$ Patients were categorised as low-, moderate- or high-risk depending on the severity of substance use. Patient sociodemographic information was collected. Immediately following screening, patients received a brief intervention (based on motivational interviewing) from the social worker. Motivational interviewing provides information or advice, motivates a change in substance use and teaches skills to reduce substance use by behavioural change. ${ }^{4}$ High-risk participants were referred to specialist substance-abuse treatment centres following intervention, as they were more likely to have substance dependence or related health conditions. ${ }^{5}$

An uncontrolled one-group pre- and post-test outcomes evaluation was performed by an independent external evaluator. All enrolled participants were evaluated. At the 3-month follow-up, the ASSIST was re-administered to all patients and a feedback questionnaire was completed.

\section{Outcomes}

\section{Primary outcome: substance use}

The ASSIST $^{5}$ - validated in several developing countries - was administered to assess the extent of problematic substance use. A substance-use involvement score was calculated for each substance used in the preceding 3 months. In the case of multiple substances, only the highest score was included in the total score.

\section{Secondary outcome: service satisfaction}

Questions were developed to assess service satisfaction and investigate potential barriers to treatment. Reasons for not accessing further treatment were explored in patients referred elsewhere for further treatment.

Analysis: $\chi^{2}$ statistics and $t$-tests were used to determine loss to follow-up. Change in substance-use involvement scores from the preto post-test were evaluated using paired-sample $t$-tests.

\section{Results}

A total of 127 substance-use patients received an intervention; 88\% were male, $78 \%$ were single, and average age was 30 years. Black 
Table 1. Pre-post differences in substance use ${ }^{*}$

\begin{tabular}{|c|c|c|c|c|c|c|}
\hline & \multicolumn{2}{|c|}{ Pre } & \multicolumn{2}{|c|}{ Post } & \multicolumn{2}{|c|}{ Comparison (pre-post) } \\
\hline & Mean & SD & Mean & SD & Mean difference & $p$-value \\
\hline Total substances $(N=92)$ & 37.60 & \pm 8.43 & 17.01 & \pm 17.19 & 20.41 & $<0.001$ \\
\hline Alcohol $(n=25)$ & 34.52 & \pm 8.82 & 10.56 & \pm 13.58 & 20.71 & $<0.001$ \\
\hline Dagga $(n=26)$ & 36.63 & \pm 9.33 & 13.77 & \pm 17.06 & 19.57 & $<0.001$ \\
\hline Mandrax and dagga $(n=9)$ & 36.78 & \pm 3.73 & 20.11 & \pm 16.78 & 16.67 & $<0.030$ \\
\hline Cocaine $(n=2)$ & 33.00 & \pm 15.56 & 28.50 & \pm 0.71 & 4.50 & $<0.742$ \\
\hline Amphetamine $(n=22)$ & 40.18 & \pm 6.63 & 23.18 & \pm 18.75 & 17.00 & $<0.001$ \\
\hline Opioids $(n=8)$ & 45.75 & \pm 5.65 & 20.88 & \pm 18.61 & 24.87 & $<0.010$ \\
\hline
\end{tabular}

African and coloured participants comprised $47 \%$ each. The primary substances of abuse were methamphetamine (30\%), alcohol (26\%), cannabis (26\%), mandrax (9\%) and opioids (7\%). Multiple substance use was reported by $44 \%$ of participants. Of the 127 patients, $68 \%$ received screening, a brief intervention and referral for specialist treatment for substance abuse; $32 \%$ received screening and a brief intervention without referral.

Ninety-two $(72 \%)$ participants completed the 3-month followup interview. Attrition was not significantly associated with sociodemographic or substance-related variables. Substance-use involvement scores decreased significantly following intervention (pre-intervention mean $37.60 \pm 8.433$, post-intervention mean $17.02 \pm 17.19, t(72)=10.89, p<0.001)$. Reductions were observed in the use of all classes of drugs except cocaine ( $p=0.742$, Table 1 ).

Of the 92 patients who completed the follow-up, 60 (66\%) were referred for further treatment, and $30(50 \%)$ received additional services. Many participants did not provide reasons for not attending further care. Others reported that they did not require further treatment or it was a waste of time $(n=8)$, it was too far to travel $(n=2)$, they had started working $(n=2)$ or they had moved away from the services $(n=1)$. Of those that attended treatment services, $55 \%$ felt that the facility met their needs, $27 \%$ that the facility mostly met their needs, and $18 \%$ that the facility did not meet their needs.

\section{Discussion}

This evaluation yielded 3 important outcomes. Firstly, it demonstrated the feasibility and acceptability of introducing SBIRT for substance use into public hospitals in South Africa, with minimal requirements for additional resourcing and little burden on health professionals.

Secondly, it provided preliminary evidence of the effectiveness of SBIRT in reducing illicit drug use in South Africa. Specifically, the intervention was successful in helping high-risk users significantly reduce their substance use to moderate levels. This adds to the limited body of knowledge supporting the effectiveness of SBIRT for illicit drug use.,4

Thirdly, the profile of people using the SBIRT programme differed from those attending specialist substance-abuse treatment facilities in the province. While there were almost an equal proportion of black and coloured respondents who received substance-use services through the programme, black South Africans comprised only $13 \%$ of treatment admissions in specialist substance-abuse facilities in $2010 .^{6}$ This suggests that there are fewer barriers to accessing substance-abuse services co-located within the public health system for black South Africans compared with stand-alone drug treatment facilities. Consequently, introducing and scaling up the provision of substance-use intervention services in the public health system may address disparities in access to services.

These findings should be interpreted with caution; there was no fidelity monitoring of the intervention and data were uncontrolled. It remains to be shown whether SBIRT services can improve medical conditions exacerbated by substance use, alleviate the abuse of prescription drugs or lower the burden on national healthcare. Longitudinal studies of hospital-based SBIRT programmes are required for this purpose.

Nevertheless, our findings suggest that hospital-based SBIRT holds promise for reducing substance use and may expand access to care for clients who have historically faced barriers to drug treatment access. We recommend expanding this pilot programme to other district hospitals in the Western Cape. Furthermore, we recommend that the centre expands its services to include strategic screening for substance use throughout the hospital (e.g. in trauma units), rather than waiting for patient referral. This could facilitate earlier detection and intervention in substance-related problems before they become too complex to treat. Furthermore, the centre should invest in the ongoing monitoring of its services to ensure quality and effectiveness of care.

Acknowledgements. We thank and acknowledge the dedication and commitment of the Jooste substance-use centre team; Maralise van Coller (social worker), Andrit Jeneke (social auxiliary worker) and ChantalLee Bailey (receptionist). We thank the patients for participating, the hospital staff and management at Jooste for their support, Dr Hering who recognised the need for this programme and the Mental Health Programme for their collaboration with Social Development. This study was funded by the Department of Social Development of the Western Cape Provincial Government, and managed by SANCA.

\section{References}

1. Stein DI, Seedat S, Herman A, Moomal H, Heeringa SG, Kessler RC, et al. Lifetime prevalence of psychiatric disorders in South Africa. Br J Psychiatry 2008:192(2):112-117. [http://dx.doi.org/ 10.1192/bjp.bp.106.029280] disorders in South Africa. BrJ Psychiatry 2008;192(2):112-117. [http://dx.doi.org/ 10.1192/bjp.bp.106.029280
Myers B, Louw J, Pasche S, Inequitable access to substance abuse treatment services in Cape Town South Africa. Subst Abuse Treat Prev Policy 2010;5:28. [http://dx/doi.org/10.1186/1747-597X-5-28] 3. Drummond C, Coulton S, James D, Godfrey C, Parrott S, Baxter I, et al. Effectiveness and costeffectiveness of a stepped care intervention for alcohol use disorders in primary care: pilot study. $\mathrm{Br}$ J Psychiatry 2009;195(5):448-456.

4. Babor TFMBG, Kassebaum PA, Grimaldi PM, Ahmed K, Bray J. Screening, brief, intervention, an referral to treatment (SBIRT). Subst Abus 2007;28(3):7-30. [http://dx.doi.org/10.1300//465v28n03_03] . WHO ASSIST Working Group. The alcohol, smoking and substance involvement screening test (ASSIST): development, reliability and feasibility. Addiction 2002;97(9):1183-1194. [http://dx/doi. org $/ 10.1046 / j .1360-0443.2002 .00185]$

6. Dada S, Parry C, Pluddemann A, Bhana A, Bachoo S, Fourie D. Alcohol and Drug Abuse Trends: July December 2010 (Phase 29) Cape Town: Medical Research Council, 2011.

Accepted 9 March 2012. 